\title{
ANÁLISIS DE LA EFICACIA INSTITUCIONAL ANTE INUNDACIONES EN EL MUNICIPIO DE TOTANA (MURCIA)
}

\author{
Francisco López Martínez \\ Universidad de Murcia
}

\section{RESUMEN}

Las herramientas de ordenación territorial constituyen el primer sistema defensivo ante los riesgos de inundación con menor impacto sobre el entorno y coste económico para salvaguardar la integridad de las personas. Con el paso del tiempo, se ha producido un progresivo incremento de la consideración de los riegos naturales, en especial los de inundación, como un criterio determinante de cara a la futura asignación de usos del suelo.

La reciente inclusión de estrictos condicionantes relacionados con la prevención de riesgos naturales en diversas normativas sectoriales, así como de herramientas de simulación capaces de predecir los efectos de los mismos, representan el nuevo estado tendencial basado en el fomento de mecanismos pasivos de prevención antes que estructurales.

Dentro del entramado de administraciones públicas encargadas de regular la ordenación territorial, los municipios, debido a su condición de instituciones que están en contacto directo con el territorio, constituyen el principal protagonista capaz de imbricar la ordenación territorial con la prevención de riesgos, motivo por el que se ha pretendido valorar en el presente trabajo el papel realizado por el consistorio de Totana, caso particular que guarda similitudes en todo el territorio nacional.

Palabras clave: riesgo, inundación, ordenación del territorio, modelización, cartografía de riesgo.

\section{ABSTRACT}

\section{Analysis of institutional effectiveness to floods in Totana's town (Murcia)}

The spatial planning tools are the first defense system against floods with less environment impact and lowest cost to safeguard the integrity of people. Over time, the natural hazards have been considered, specially floods, like a determinant standard for the future land-use planning.

Recently, the apparition of related conditions with the prevention of natural hazards in various sectoral laws, also simulations tools capable of predicting their effects, are the new paradigm based on passive prevention mechanisms before the structural responses.

Inside the different public administration responsible spatial planning, the municipals institutions, because they are the organism who are in direct contact with territory, they are the main character able to imbricate the spatial planning with the risk prevention, reason because the paper of the Totana's hall town has been evaluated in this work, particular case but with similarities in all the Spanish territory.

Keywords: risk, flood, spatial planning, modeling, cartography of risk.

\section{INTRODUCCIÓN}

Las inundaciones ocasionadas por procesos fluviales constituyen el peligro de origen natural más recurrente en los entornos mediterráneos (Camarasa y Soriano, 2012). Su coste y repercusión, tanto en volumen de pérdidas económicas como sociales, se erige como el más elevado de todos los riegos que afectan al ser humano (Annual Disaster Statistical Review, 2012).

Los procesos de ocupación de áreas inundables, en ocasiones por desconocimiento pero mayoritariamente por negligencia, han provocado la rotura de la estrecha conexión asentamiento-recurso hídrico, transformándola en el dualismo población-inundación. A lo largo de la historia las sociedades han

Contacto: $\underline{\text { lm5@um.es }}$ 
intentado reducir la severidad e intensidad de los fenómenos de inundación mediante el desarrollo de numerosas y costosas medidas estructurales cuyos resultados, tal y como demostró la paradoja hidráulica expuesta por White et al. (1958) y White (1975), no han sido siempre los deseados.

Durante los últimos años, a pesar del extenso conocimiento desarrollado en torno a la componente física que rige los procesos naturales, la especulación del recurso suelo y la falsa sensación de seguridad provocada por las infraestructuras hidráulicas, han acentuado la componente social presente en la ecuación del riesgo, la vulnerabilidad, provocando un incremento de la acción y efecto de los riesgos de origen natural sobre las sociedades. A pesar de que no existe unanimidad del número de dimensiones en que puede desglosarse la vulnerabilidad, pues según el autor y la obra consultada pueden encontrarse desde once (Wilches-Chaux, 1993), nueve (Wilches-Chaux, 1989) u ocho (Parker et al., 2009), hasta cinco (Smith y Petley, 2009), tres (Cannon, 1994; Anderson y Woodrow, 1989) o dos (Bohle, 2001), constituye un hecho aceptado que la vulnerabilidad como concepto global está compuesta un número de factores o vulnerabilidades individuales cuyo comportamiento particular puede influir de manera sinérgica o antagónica sobre el resto (figura 1).

Figura 1. Diferentes factores que componen la vulnerabilidad global.

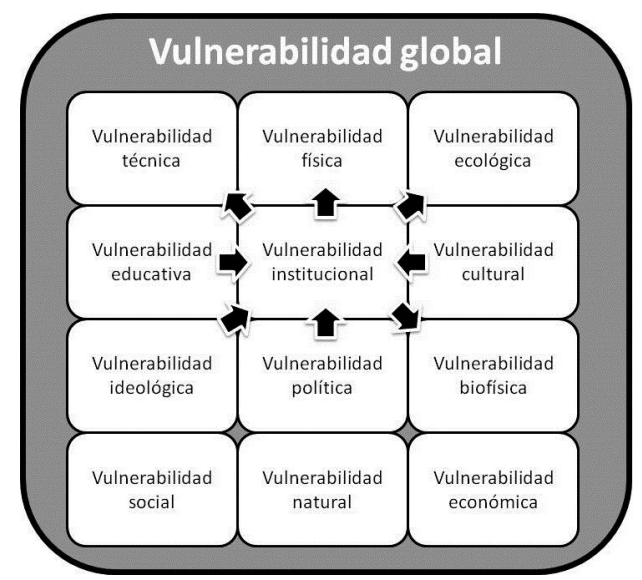

Las flechas indican las diferentes interacciones positivas existentes entre la vulnerabilidad institucional y el resto.

Fuente: Elaboración propia.

Independientemente del número de facetas que componen la vulnerabilidad global, un papel fundamental de la misma está interpretado por las diferentes administraciones encargadas de promulgar, gestionar y aplicar los condicionantes en materia de prevención de riesgos de origen natural, la denominada como vulnerabilidad institucional (Wilches-Chaux, 1993; Parker et al., 2009). Debido a la organización político-administrativa interna de España, así como la carencia de una ley marco sobre riegos naturales, diversas son las administraciones responsables de compaginar e imbricar la prevención de riesgos con las distintas normativas de incidencia sectorial (suelo, agua, medio ambiente, recursos naturales, etc...), normativas donde las relacionadas con la ordenación territorial, agua y protección civil han adquirido un protagonismo esencial ante el riesgo de inundaciones (Olcina, 2004).

La ordenación territorial, marco regulador de los instrumentos de planeamiento y "reflejo palpable del grado de eficiencia y equidad adquirido por una determinada sociedad" (Sáenz de Buruaga, 1980: 18), debe considerar ineludiblemente los rasgos físicos del territorio (Olcina y Giménez, 2004) e integrar durante su redacción el análisis de riesgos, pues constituye la metodología adecuada para su identificación, evaluación, mitigación y toma de decisiones (Ayala-Carcedo, 2002). El conjunto de condicionantes normativos establecidos en los instrumentos de ordenación, previa materialización sobre el territorio, son delimitados gráficamente a través de los planos de ordenación (art. 121 Decreto Legislativo Regional 1/2005), documento básico de todo PGMO encargado de reflejar espacialmente, entre otros, los usos asignados al suelo.

Dentro de toda la batería de riesgos con origen natural que afectan al territorio español (terremotos, sequías, inundaciones, vulcanismo, erosión, etc...), la importancia territorial y socio-económica de los riesgos de inundaciones (González, 1988; Camarasa, 2002; Pujadas, 2002; Ayala-Carcedo, Olcina y Vilaplana, 2003; Consorcio de Compensación de Seguros, 2008; Llorente et al., 2009) ha motivado que los instrumentos de ordenación territorial cuenten con un soporte cartográfico de los distintos niveles 
de riesgo, los mapas de áreas inundables (Directriz Básica de Inundaciones, 1995; Sistema Nacional de Cartografía de Zonas Inundables, 2013), documentos predictivos básicos para conocer los espacios tradicionalmente afectados por el peligro de inundación.

La mencionada cartografía se ha convertido en una herramienta imprescindible para el análisis y evaluación de los riesgos presentes en el ámbito de ordenación (Darío, 1993), pues abarca "desde las etapas iniciales como fuente de información, pasando por el análisis como soporte de modelización, y finalizando con la edición de resultados como base de representación" (Diez y Pujadas, 2002: 997). Sin embargo, a pesar de la existencia de un cuerpo legislativo compuesto por diferentes normativas estatales y autonómicas en pro de la utilización de dicha cartografía (Olcina, 2004), así como por los propios condicionantes plasmados en multitud de herramientas de planeamiento local, sus premisas no han sido siempre consideradas, lo que se traduce en un incremento de la superficie expuesta y pone de manifiesto que "el riesgo de inundación nunca desaparece completamente sino que adopta nuevas formas y afecta a nuevos territorios en respuesta a dinámicas socioterritoriales cambiantes" (Saurí et al., 2010: 270).

En consecuencia, el análisis de los distintos instrumentos de planeamientos local permite determinar el grado de eficacia institucional adquirido de manera autónoma por un municipio determinado. A lo largo del presente trabajo se evaluará cómo ha considerado el consistorio de Totana (Murcia) en su máximo exponente de ordenación municipal, el Plan General Municipal de Ordenación (PGMO), los riesgos derivados de los procesos de inundación, para posteriormente comprobar la eficiencia de las medidas de carácter preventivo.

El hecho de haber elegido el municipio de Totana radica en la intrínseca conexión existente entre el casco principal del término, Totana, y un curso fluvial de marcado carácter torrencial, la Rambla de La Santa, cuyo peculiar comportamiento hidrológico se encuentra supeditado, al igual que la mayoría de cursos intermitentes distribuidos a lo largo de todo el sureste español, a las precipitaciones extraordinarias de gran intensidad, culpables de prácticamente la mayor parte de inundaciones sufridas en la España Levantina (Camarasa, 2002). Además, aprovechando que la futura ordenación territorial del municipio se encuentra ampliamente desarrollada en el vigente PGMO que todavía no ha sido materializado, se podrán detectar, con carácter "pre-desastre", las carencias o incongruencias en materia de actuaciones preventivas no estructurales ante el riesgo de inundación.

\section{METODOLOGÍA Y FUENTES}

Atendiendo a todo lo anterior, entre otros casos en los que también puede emplearse, se ha optado por utilizar la cartografía oficial de consulta (Olcina, 2010; Pérez, 2012) aplicable durante el proceso de redacción y de desarrollo del PGMO, herramienta de planeamiento que rige la actual ordenación municipal. La cartografía de consulta, anteriormente entendida como mapas de áreas potencialmente inundables (Díez y Pujadas, 2002) pero actualmente considerada como de áreas inundables (Pujadas et al., 1997), ha sido proporcionada por la Dirección General de Protección Civil de la Región de Murcia y el Ministerio de Agricultura, Alimentación y Medio Ambiente (MAGRAMA), cuyos resultados se encuentran representados, respectivamente, en el Plan Especial de Protección Civil Ante el Riesgo de Inundaciones de la Región de Murcia (Plan INUNMUR) y el Sistema Nacional de Cartografía de Zonas Inundables (SNCZI).

Figura 2. Esquema teórico para la elaboración de mapas de riesgo de daños inundación.

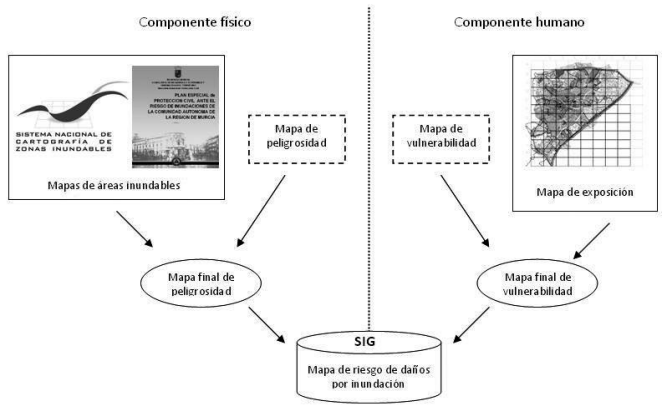

Fuente: Adaptado de Ribera Masgrau (op. cit.)

La integración en un SIG de todos los factores anteriores (figura 2), por un lado, las figuras de planeamiento, encargadas de determinar la exposición y, por otro, la cartografía de áreas inundables, expresión 
del área potencialmente afectada por las inundaciones, devuelve una visión o un mapa simplificado pero conciso (a falta de considerar la peligrosidad y vulnerabilidad), de los riesgos de daños por inundación (Ribera, 2004). A partir de los resultados proporcionados por dicho mapa, el regidor municipal, así como cualquier otro actor interviniente en el proceso planificador, puede y debe configurar los usos del suelo en función de aquellos sectores más idóneos, o lo que es lo mismo, con menor riesgo de inundación, salvaguardando del proceso urbanizador las zonas más susceptibles de sufrir esta tipología de eventos.

\section{3. ÁREA DE ESTUDIO}

La Rambla de la Santa, también conocida como Rambla de Totana, representa el típico curso fluvial mediterráneo cuyo cauce se encuentra seco durante prácticamente la totalidad del año, sin embargo, debido al carácter torrencial de las precipitaciones en la región Mediterránea, es capaz de evacuar grandes caudales en breves intervalos de tiempo (Pulido, 1993). La cuenca hidrográfica de la Rambla se encuentra situada en el SW de la Región de Murcia, en pleno corazón del Bajo Guadalentín ${ }^{1}$, comprende una extensión aproximada de $60 \mathrm{~km}^{2}$ y abarca parte de los municipios de Aledo y, en mayor proporción, Totana (figura 3).

Figura 3. Localización de la Rambla (izquierda) e imagen tridimensional de su cuenca (derecha).
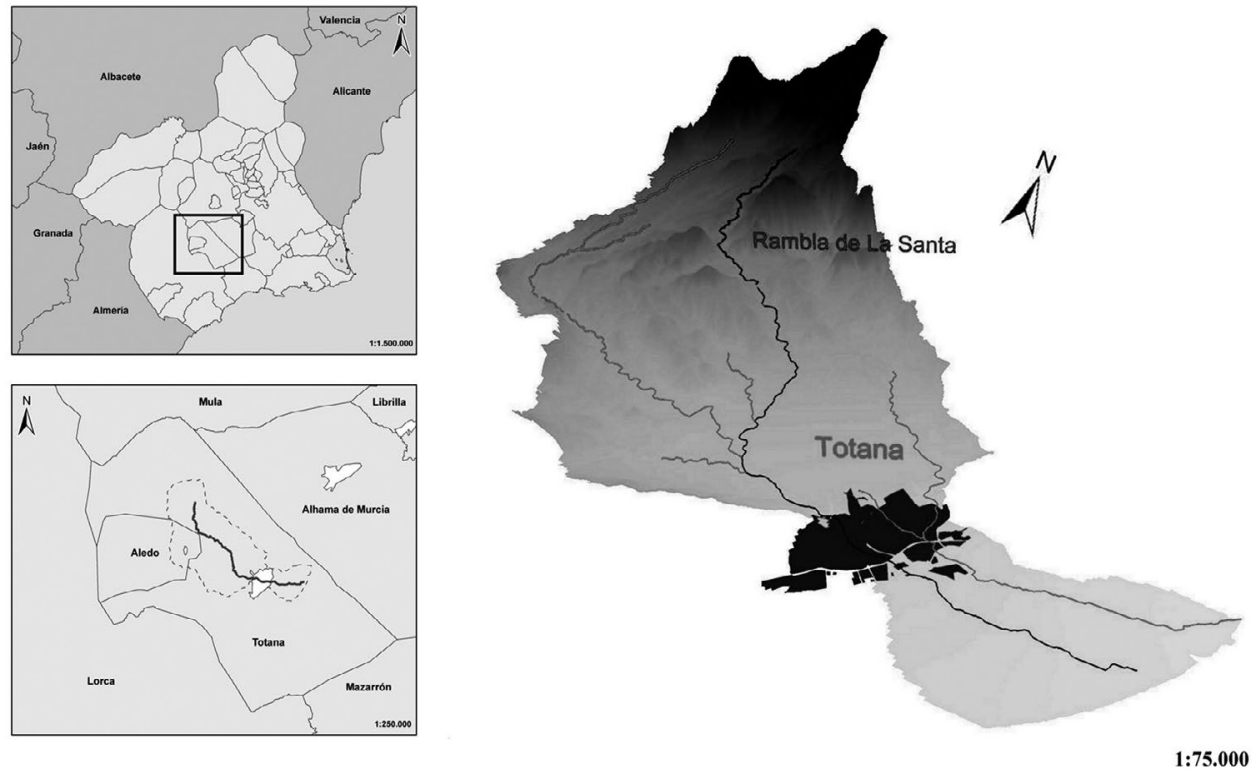

Fuente: Instituto Geográfico Nacional y Confederación Hidrográfica del Segura. Elaboración propia.

El curso de la rambla posee su nacimiento a unos $1.280 \mathrm{~m}$. de altitud, en las laderas de Sierra Espuña, al SE del Collado Bermejo, en un paraje conocido como La Santa (topónimo del que recibe su denominación). El recorrido de la Rambla discurre de manera paralela a una falla neotectónica de segundo orden, desaguando a unos $17 \mathrm{~km}$. aguas abajo de la localidad de Totana, en el Valle del Guadalentín. A pesar de la escasa extensión de la cuenca hidrológica, el curso principal de la Rambla presenta una intensa actividad, pues durante su recorrido recibe por ambos márgenes el caudal proveniente de diversos afluentes como la Rambla de Yéchar, el Barranco de Ballesteros por la izquierda y el Barranco del Estrecho del Arco, la Rambla de los Molinos y la de los Bueyes por la derecha. Sin embargo, a pesar de todos los aportes hídricos que recibe la Rambla, la potencia de su caudal suele ser tan escasa que le imposibilita alcanzar el nivel de base del Río Guadalentín y dispersa su contenido en forma de abanico aluvial en la huerta de Totana, aguas abajo del municipio.

Debido a la morfología del terreno, bastante escarpada por su cercanía a Sierra Espuña, así como por la dureza de los materiales litológicos existentes, elementos del complejo Maláguide, el encajamiento de

1 Debido a la multitud de divisiones comarcales existentes para la Región de Murcia, en el presente trabajo se ha seguido la delimitación establecida por el ya extinguido Consejo Regional de Murcia, fundamentalmente redactado por Fuentes y Calvo (1982). 
la red fluvial de la Rambla se ha producido a lo largo de un espacio confinado cuya única desembocadura hacia el Valle del Guadalentín está inserta en una cubeta de recepción delimitada por dos elevaciones neógenas localizadas a ambos lados de la localidad de Totana y que han servido como barrera natural para la retención de los materiales cuaternarios de las elevaciones montañosas cercanas. Las peculiaridades orográficas de la zona, además de condicionar el comportamiento y distribución de los elementos naturales, han marcado los usos del medio, estableciendo tanto el emplazamiento de la huerta de Totana, desarrollada en la propia cubeta de recepción debido a los suelos fértiles, fáciles de trabajar y con un suministro de agua proveniente de las elevaciones, como el asentamiento de la propia localidad, al pie de las dos elevaciones neógenas anteriormente indicadas.

Históricamente, la expansión del núcleo urbano de Totana se ha realizado en los márgenes del cauce de la Rambla de La Santa, provocando la división de la localidad en dos grandes barrios: Triana al NE y Sevilla al SW. En la actualidad, debido a que la Rambla constituye parte del entramado urbano de la localidad, se ha incrementado considerablemente la exposición de la población a la recurrencia y severidad de los fenómenos de inundación, causando la aparición de un nuevo componente de la ya citada vulnerabilidad global, la vulnerabilidad biofísica (Wilches-Chaux 1993, 1989; Cutter et al., 2003).

Ante la carencia de algún tipo de catálogo local acerca de los eventos de inundación sufridos por la localidad que confirme lo anterior, se ha elaborado un registro histórico de los mismos (gráfico 1) tomando como base la información recogida en el Catálogo Nacional de Inundaciones Históricas (CNIH) editado por Protección Civil (2006), los acontecimientos reflejados en el capítulo Las inundaciones del Atlas Global de la Región de Murcia (2007), los datos proporcionados por Confederación Hidrográfica del Segura (CHS), IGME (2007), Pelegrín (2006), Romero y Maurandi (2000), Romero (2007), así como las hemerotecas de los dos principales periódicos regionales (La Verdad y La Opinión).

Gráfico 1. Frecuencia de las inundaciones registradas en la localidad de Totana.

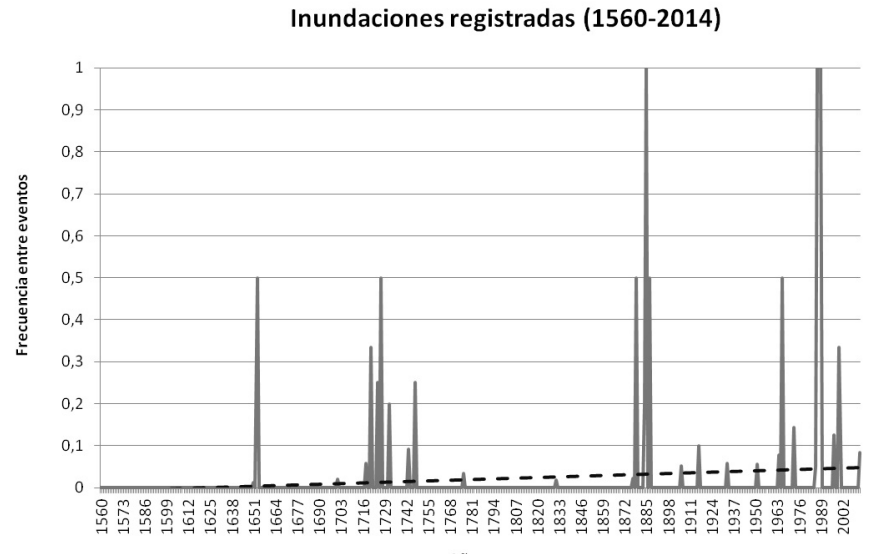

Año

El valor de cada pico representa la inversa de los años transcurridos desde el último evento registrado y la línea discontinua la tendencia marcada por el histórico de inundaciones.

Tal y como puede observarse en el gráfico anterior, durante, aproximadamente, las últimas cinco centurias la localidad se ha visto afectada por más de una treintena de sucesos de inundación cuyo principal agente causal ha sido la Rambla de La Santa (60 \% de los casos). El período del año en el que se han registrado un mayor número de sucesos coincide con el final de la época otoñal e inicio de la invernal (47\% de los eventos), etapa que es seguida muy de lejos con el comienzo del estío (16\%).

A raíz del último y dramático episodio de inundación sufrido en 1964, Confederación Hidrográfica del Segura (CHS) optó por canalizar el cauce de la Rambla a lo largo del considerado por entonces como casco urbano de la localidad, construyendo un canal de 1.064 metros de longitud con una capacidad media de desagüe de $420 \mathrm{~m}^{3} / \mathrm{s}$. Hasta el momento, el acondicionamiento del cauce, ejemplo inequívoco de la adopción de medidas preventivas debido al aumento de la vulnerabilidad biofísica, ha cumplido su propósito, pues incluso con el episodio vivido el pasado 28 de septiembre de 2012 cuando se llegaron a registrar precipitaciones acumuladas de $115 \mathrm{~mm}$ en una estación de la AEMET y $117^{\prime} 8 \mathrm{~mm}$ en otra particular, la Rambla no llegó a desbordarse. Sin embargo, la reciente expansión de la localidad ha desplazado los procesos de inundación hacia otras áreas, los cauces de la Rambla de las Peras (S) y de Los $\operatorname{Arcos}(\mathrm{N})$, 
situadas respectivamente al Sur y al Norte del municipio. Ambos cursos de agua torrencial carecen de una batería de medidas correctoras suficientemente desarrolladas, sobre todo para la rambla de Los Arcos, cuyo trayecto, a pesar de encontrarse encauzado durante un tramo aproximado de $220 \mathrm{~m}$., atraviesa al mismo nivel el viario de la localidad (figura 4 derecha).

Figura 4. Localización de la ya inexistente placa de inundación conmemorativa de la altura que alcanzó el agua durante la riada del 27 de junio de 1887 ( $\approx 3,5 \mathrm{~m}$.), riada de San Zoilo (izquierda), y cruce del cauce de la rambla de los Arcos al mismos nivel que el viario de la localidad (derecha).

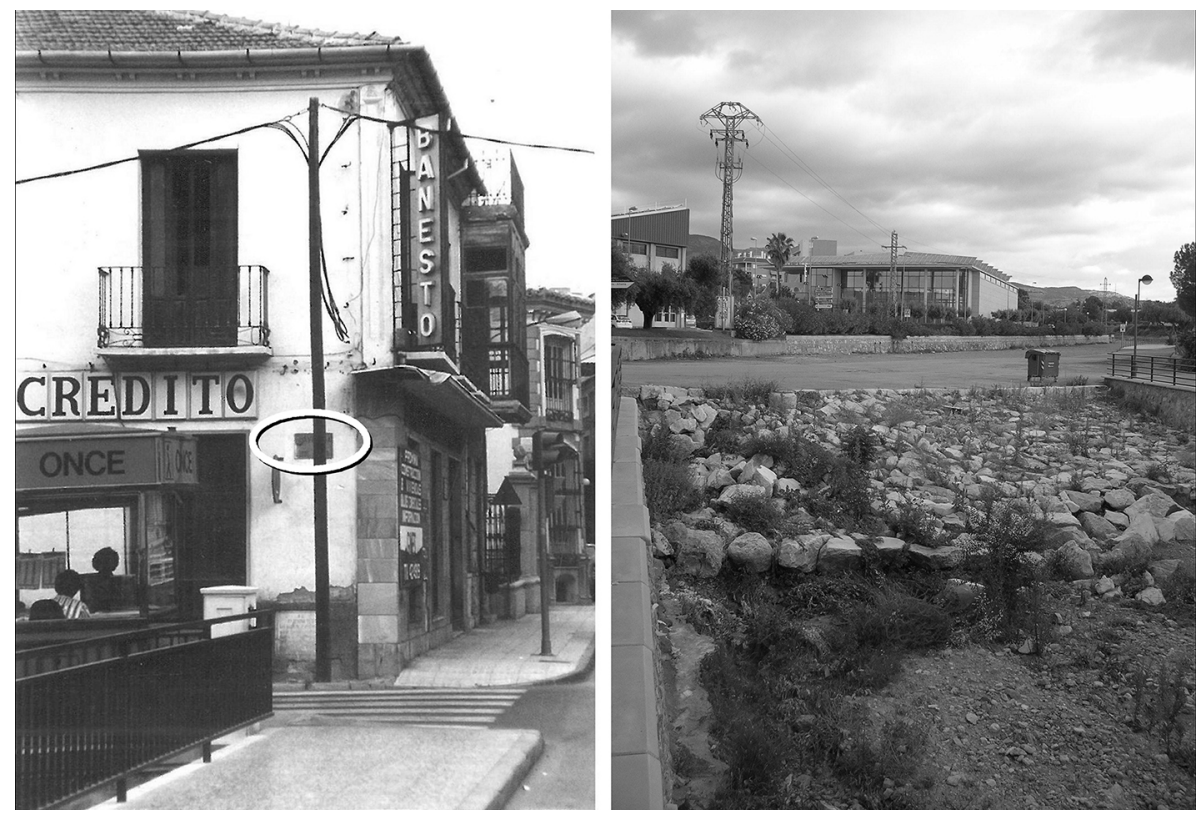

Fuente: Extraído de Pelegrín (op. cit.) y datos obtenidos sobre el terreno.

\section{ORDENACIÓN DEL TERRITORIO Y GESTIÓN DE RIESGOS A ESCALA LOCAL. EL MUNICIPIO DE TOTANA}

El planeamiento urbanístico o la ordenación territorial, en función de la escala de trabajo considerada, constituye la principal herramienta jurídica que posee en su haber el gobierno regional y municipal para determinar sus políticas de crecimiento y estadios de desarrollo, convirtiéndose en "la medida de reducción del riesgo más racional y menos agresiva sobre el medio" (Olcina, 2004: 72). Además de reflejar fielmente el grado de percepción y sensibilidad institucional ante el peligro por inundación, las herramientas de planificación constituyen el máximo exponente de cómo la interacción antrópica sobre los usos del territorio pueden acelerar o intensificar su impacto (González, 2002).

Diversas son las esferas administrativas con competencias en materia de ordenación territorial cuyos márgenes de actuación están limitados a los postulados establecidos por los estamentos superiores. La jerarquía existente, tanto a nivel normativo como competencial, concede a cada escalón de gobierno la posibilidad de concretar y adaptar los condicionantes normativos a las particularidades territoriales que caracterizan el área objeto de ordenación.

El PGMO representa el instrumento central de ordenación municipal encargado de plasmar sobre el territorio los diferentes condicionantes establecidos en materia de usos de suelo. El cometido de todo Plan radica en determinar la clasificación ${ }^{2}$ del suelo, definir los elementos esenciales de la estructura general y orgánica del territorio, precisar el modelo de ciudad y de los asentamientos urbanos, decidir los criterios para su desarrollo y determinar los espacios y elementos de especial protección. Además, con la

2 Según Beltrán (2006), en ocasiones, los conceptos de clasificación y calificación del suelo suelen ser utilizados de manera indistinta, sin embargo sus descripciones son completamente diferentes, pues mientras la clasificación determina el régimen urbanístico en que se divide el suelo (urbano, urbanizable o no urbanizable), la calificación se refiere a la definición de usos e intensidades de las distintas clases de suelo (sectorizado, sin sectorizar, equipamientos, etc...). 
intención de regular aspectos no previstos o insuficientemente desarrollados por el PGMO, la normativa autonómica, Real Decreto Legislativo 1/2005, concede la posibilidad de redactar Normas Complementarias de Planeamiento General (NNSS), "principales herramientas que tiene el administrador municipal para ordenar usos en el territorio y, en relación con ellos, incorporar medidas que puedan contribuir a mitigar los riesgos naturales inherentes a una localidad" (Olcina, 2004: 73).

Los instrumentos de ordenación de este tipo han encontrado un importante aliado en los mapas de riesgo, medio que permite conocer los posibles efectos de los procesos naturales sobre lo planificado y, según los mismos, determinar qué áreas presentan una mayor vulnerabilidad biofísica. Para el caso de los fenómenos de inundación, tanto el autonómico Plan INUNMUR (2007) como el nacional SNCZI (2013), constituyen la cartografía oficial de consulta que todo planificador local debe considerar e integrar durante el proceso de ordenación territorial de cualquier municipio murciano (figura 6).

Figura 5. Normativas de ordenación territorial y cartografía oficial de consulta que debe ser considerada durante la redacción de un PGMO en la Región de Murcia.

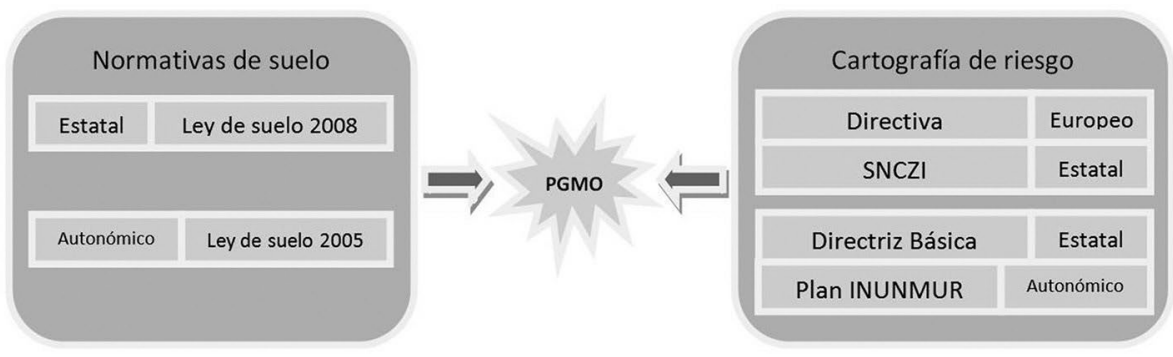

Fuente: Elaboración propia.

\subsection{La planificación urbanística de Totana. El PGMO y su consideración del riesgo de inundación}

El municipio de Totana, al igual que la mayoría de los municipios murcianos, posee como elemento central de ordenación territorial un PGMO. El Plan inició su tramitación en el año 2004, en sintonía con el espíritu urbanizador de la Ley estatal del suelo (Ley 6/1998 sobre régimen del suelo y valoraciones). Tras un largo procedimiento administrativo en el que se produjeron diversas modificaciones y subsanaciones, el Plan fue aprobado definitivamente, aunque de modo parcial, en el año 2011. Sus disposiciones sólo son aplicables al Suelo Urbano Consolidado del Núcleo (Fig. 6), careciendo de ejecutividad en el resto de sectores donde son aplicables, sin perjuicio de las modificaciones introducidas por el Plan, las NNSS vigentes desde 1981.

La primera alusión al riesgo de inundación que aparece en el Plan está relacionada con las áreas definidas entorno al margen de los cauces, estableciendo su art. 131, en consonancia con la Ley de Aguas ${ }^{3}$ y el Reglamento del Dominio Público Hidráulico ${ }^{4}$, la delimitación a ambos lados de cada cauce de dos áreas: zona de servidumbre y zona de policía, delimitadas por 5 y 100 metros lineales de anchura respectivamente.

En la zona de servidumbre quedan prohibidas las construcciones o cerramientos de cualquiera tipo, salvo los otorgados excepcionalmente por Confederación Hidrográfica del Segura. En la zona de policía, por el contrario, sí se permiten las actuaciones estructurales siempre y cuando sean compatibles con las Normas Urbanísticas de la localidad y cuenten con autorización previa de Confederación. La atribución competencial que poseen los organismos de cuenca para delimitar el Dominio Público Hidráulico, suple, en ciertas ocasiones y por mera coincidencia debido a cuestiones de proximidad, el cometido de las administraciones locales de preservar del proceso urbanizador aquellas áreas con mayor peligro de inundación:

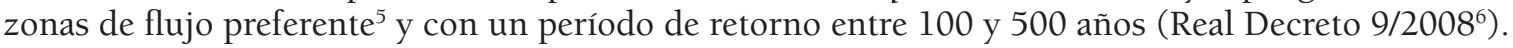

3 Real Decreto Legislativo 1/2001, de 20 de julio, por el que se aprueba el texto refundido de la Ley de Aguas.

4 Real Decreto 849/1986, de 11 de abril, por el se aprueba el Reglamento del Dominio Público Hidráulico que se desarrolla los títulos preliminar, I, IV, V, VI y VII de la Ley 29/1985, de 2 de agosto, de Aguas.

5 Tanto el RD 9/2008 como el RD 903/2010 coinciden en la definición de zona de flujo preferente, entendiéndose como el área que incluirá la vía de intenso desagüe, así como las zonas de elevada peligrosidad para la avenida de 100 años de periodo de retorno.

6 Real Decreto 9/2008, de 11 de enero, por el que se modifica el Reglamento del Dominio Público Hidráulico, aprobado por el Real Decreto 849/1986, de 11 de abril. 
Figura 6. Clasificación del suelo de Totana.

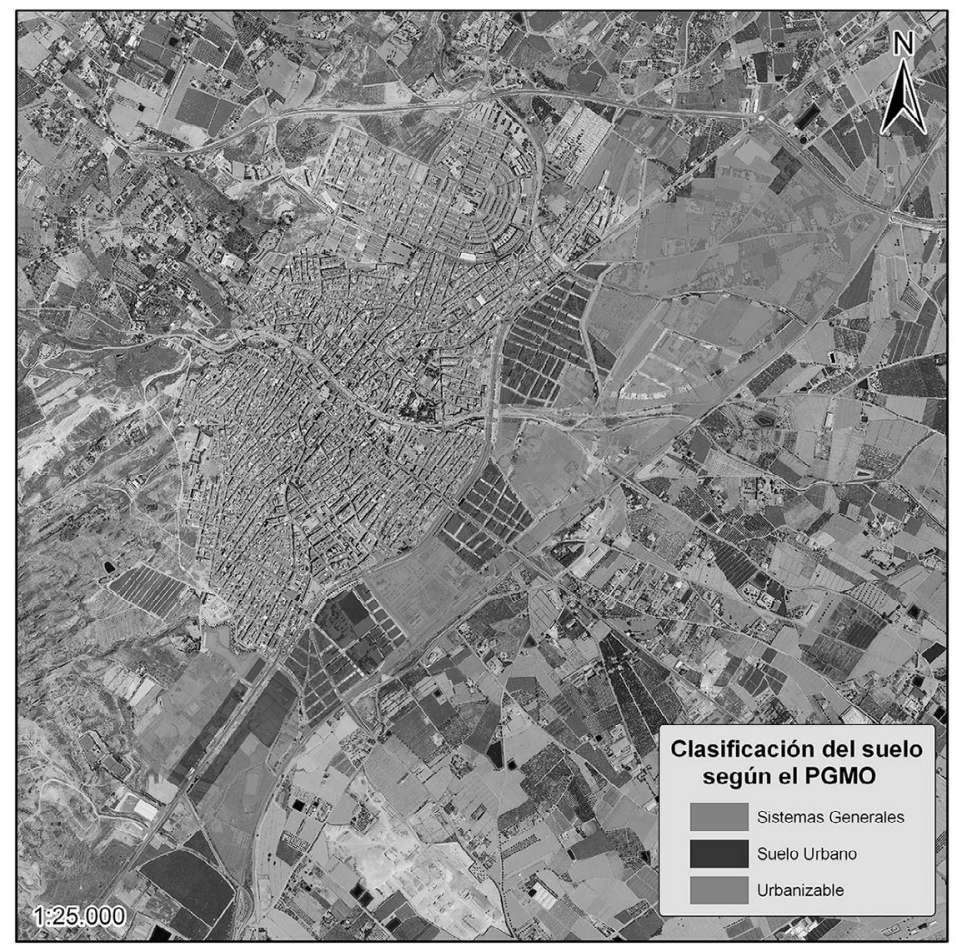

Fuente: PGMO de Totana. Elaboración propia.

Respecto a las limitaciones en materia de usos de suelo relacionadas con el peligro de inundación, las normas urbanísticas desarrollan el Plan e integran en su articulado la gestión preventiva de los riesgos de origen natural a través de la clasificación del suelo, transcribiendo prácticamente los preceptos regionales al considerar las áreas afectadas por fenómenos de inundación como "suelo no urbanizable de protección específica" (art. 213.1 del PGMO). Por otro lado, atendiendo a un concepto tan recurrente aunque de ambiguo contenido como es el desarrollo sostenible (art. 213.3 del PGMO), el Plan también contempla la conservación de ciertas áreas con un interés especial y su declaración como "suelo no urbanizable inadecuado para el desarrollo urbano", además, las áreas con presencia de ramblas y cauces están definidas por el Plan como "zonas a proteger", catalogación que las permite integrarse en la denominación de "suelo inadecuado para el desarrollo urbano" (art. 214 del PGMO).

A pesar de que el Plan establece la consideración de los riesgos de origen natural acreditados por el planeamiento sectorial como un criterio definitorio ante la futura asignación de usos al suelo, el urbanizador municipal no condicionó los usos del suelo en función del único instrumento de referencia vigente durante su redacción, el Plan INUNMUR. Según establecen las simulaciones de dicho Plan (figura 7), un $7 \%$ del suelo clasificado como urbanizable estaría afectado por fenómenos de inundación con un período de retorno de 50 años, extensión que aumentaría hasta el 9\% o el 10,5\% para intervalos de 100 y 500 años respectivamente.

En el hipotético caso de que el Plan fuera aprobado en la actualidad y considerase como cartografía de referencia el SNCZI, el escenario de riego del municipio se encuadraría en un estadio mucho más agravado, pues prácticamente un tercio de la totalidad del suelo definido como urbanizable, unas 25 ha., así como el extremo sur de la localidad y que actualmente carece de un uso de suelo, se encontraría afectado por una inundación con un período de retorno de 50 años (figura 8), situación que empeoraría considerablemente, pudiendo llegar casi a duplicar la superficie afectada ante una inundación con un T de 500 años.

\section{CONCLUSIONES}

A pesar de la histórica recurrencia de los fenómenos de inundación, el ser humano todavía no ha sido capaz de adaptar y compaginar su desarrollo, fundamentalmente el urbanístico, con el medio que 
lo rodea. Debido a este desajuste de usos territoriales, el legislador se ha encontrado ante la imperiosa necesidad de establecer una serie de medidas encaminadas a reducir a la mínima expresión esta tipología de riesgos y sus consecuencias.

Los diferentes instrumentos de ordenación territorial han intentado contrarrestar los efectos de las inundaciones a través de la introducción progresiva de limitaciones territoriales en función de su presencia. Sin embargo, a pesar de los innumerables eventos catastróficos que han azotado y asolado multitud de municipios españoles, especialmente en la vertiente mediterránea, escasas o nulas han sido las medidas de planificación no ingenieriles adoptadas en la mayoría de los desarrollos urbanísticos recientemente materializados.

Claro ejemplo de esta situación lo constituye Totana, municipio que pese a su histórica conexión con los cursos fluviales torrenciales que atraviesan la localidad, Rambla de la Santa, de las Peras y de los Arcos, no ha sido capaz de adaptar su planeamiento y ha absorbido dichos cauces dentro del entramado urbano del municipio. Esta integración de cursos hídricos de escasa envergadura ha supuesto, además del pertinente incremento de la vulnerabilidad biofísica tradicionalmente reducido a los márgenes del cauce, un traslado de los mayores niveles de riesgo frente a las inundaciones "desde las grandes cuencas fluviales a las cuencas pequeñas de comportamiento torrencial, esto es, barrancos, ramblas, rieras, arroyos" (Olcina, 2004: 64).

El detrimento de la eficacia institucional provocada por una clasificación del suelo carente de las premisas legalmente definidas en el momento de tramitación y aprobación del PGMO (Directriz, Ley del suelo nacional y regional), ha tenido un impacto directo, negativo y catalizador sobre la vulnerabilidad biofísica ante los procesos de inundación. Esta mala praxis administrativa, además de aumentar la vulnerabilidad global, también ha puesto de manifiesto la inherente importancia que posee la componente antrópica dentro del concepto de riesgo, cuya acción sobre los parámetros naturales y la incompatibilidad de actividades ha llegado a transformar un riesgo de origen estrictamente natural o primario (Ortega, 1991) en uno inducido.

En ciertas ocasiones, la autonomía que poseen los municipios españoles para aprobar instrumentos de ordenación territorial, figuras administrativas bastante controvertidas debido a los cuantiosos intereses económicos que las circundan, ha degradado y aumentado la permisividad de los condicionantes normativos en prejuicio de la seguridad ciudadana. Alcanzado este punto, debe cuestionarse, además del papel de la administración local, la pasividad negligente contraída por la administración autonómica, esfera responsable de aprobar en última instancia los instrumentos de planeamiento locales.

Por último, a pesar que el Plan no ha considerado ninguna de las cartografías oficiales de consulta sobre el riesgo de inundación citadas, afortunadamente la normativa en materia de aguas concede a los organismos de cuenca la potestad necesaria para contrarrestar la desidia contraída por los gestores locales ante la prevención de riesgos por inundación, pues a través del informe preceptivo que debe emitir para las construcciones cercanas a los cursos de agua existentes, documento con carácter de autorización, adquiere la autoridad necesaria para regular adecuadamente el desarrollo parcial del planeamiento urbano de acorde a las premisas recogidas en materia de riesgos por inundación.

\section{DISCUSIÓN}

Para el caso de las inundaciones, los instrumentos de ordenación territorial representan un eficiente sistema defensivo capaz de amoldar los distintos usos del suelo en función de las características biofísicas del ámbito de ordenación. Esta herramienta preventiva pasiva no estructural constituye la máxima expresión de la gestión integrada del riesgo que permite armonizar, a través de condicionantes y limitaciones, las distintas etapas de desarrollo e infraestructuras urbanas localizadas en áreas susceptibles de fenómenos de inundación. Sin embargo, en diversos municipios ligados a cursos hídricos, como ocurre en Totana, las influencias político-económicas, la relativa flexibilidad legislativa, así como el grado de concienciación del redactor del planeamiento, pueden reducir drásticamente la fortaleza de las figuras de ordenación territorial como medidas preventivas, dando lugar a un aumento de la exposición y, por extensión, del riesgo tras integrar en el planeamiento local cursos hídricos de escasa envergadura pero de comportamiento torrencial. Por otro lado, dichas figuras no deben reducirse o entenderse como meras herramientas diseñadas para constreñir el proceso urbanizador en función del riesgo de inundación, sino como un reflejo adaptativo al entorno que permite compaginar y optimizar los distintos usos del suelo. 


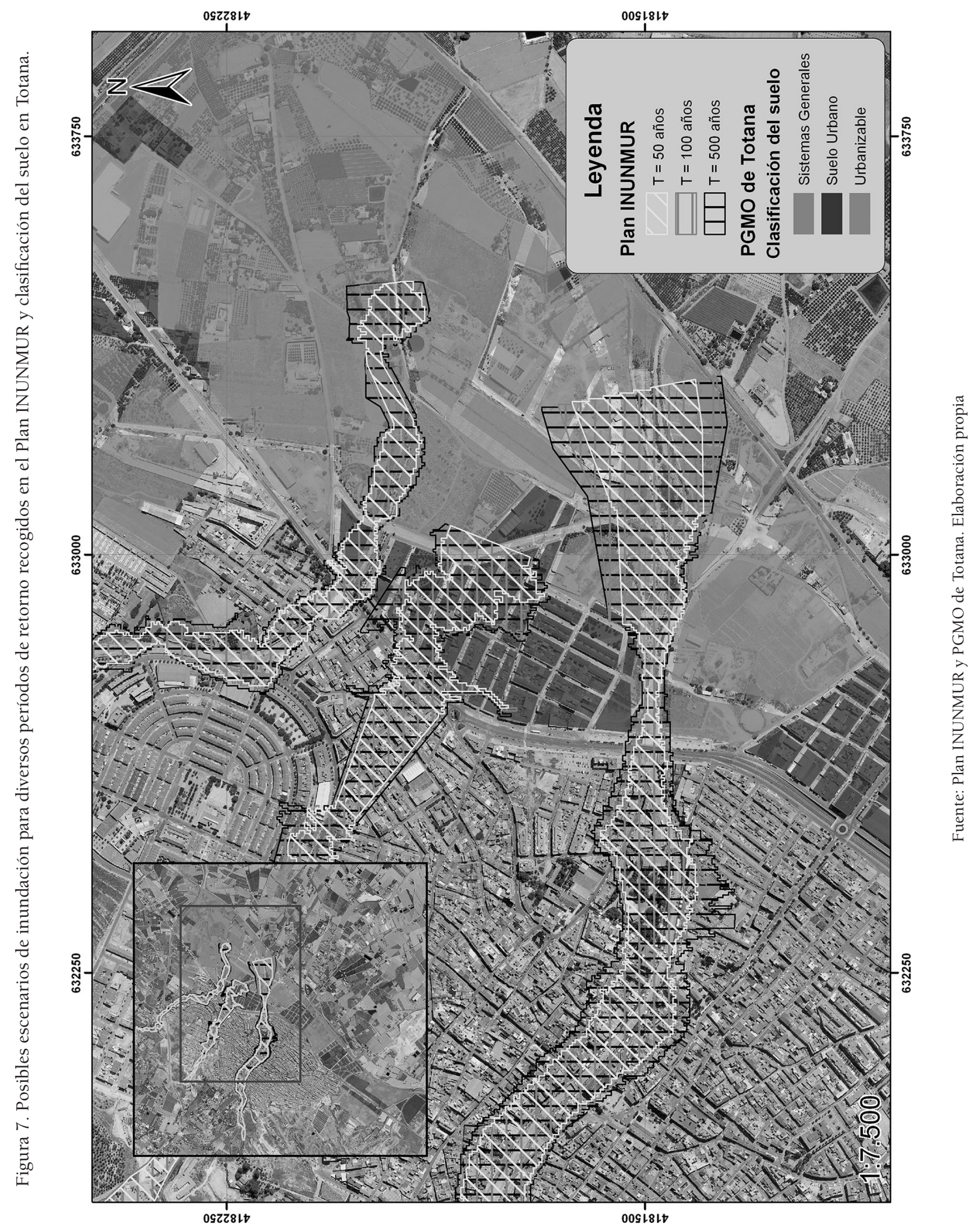




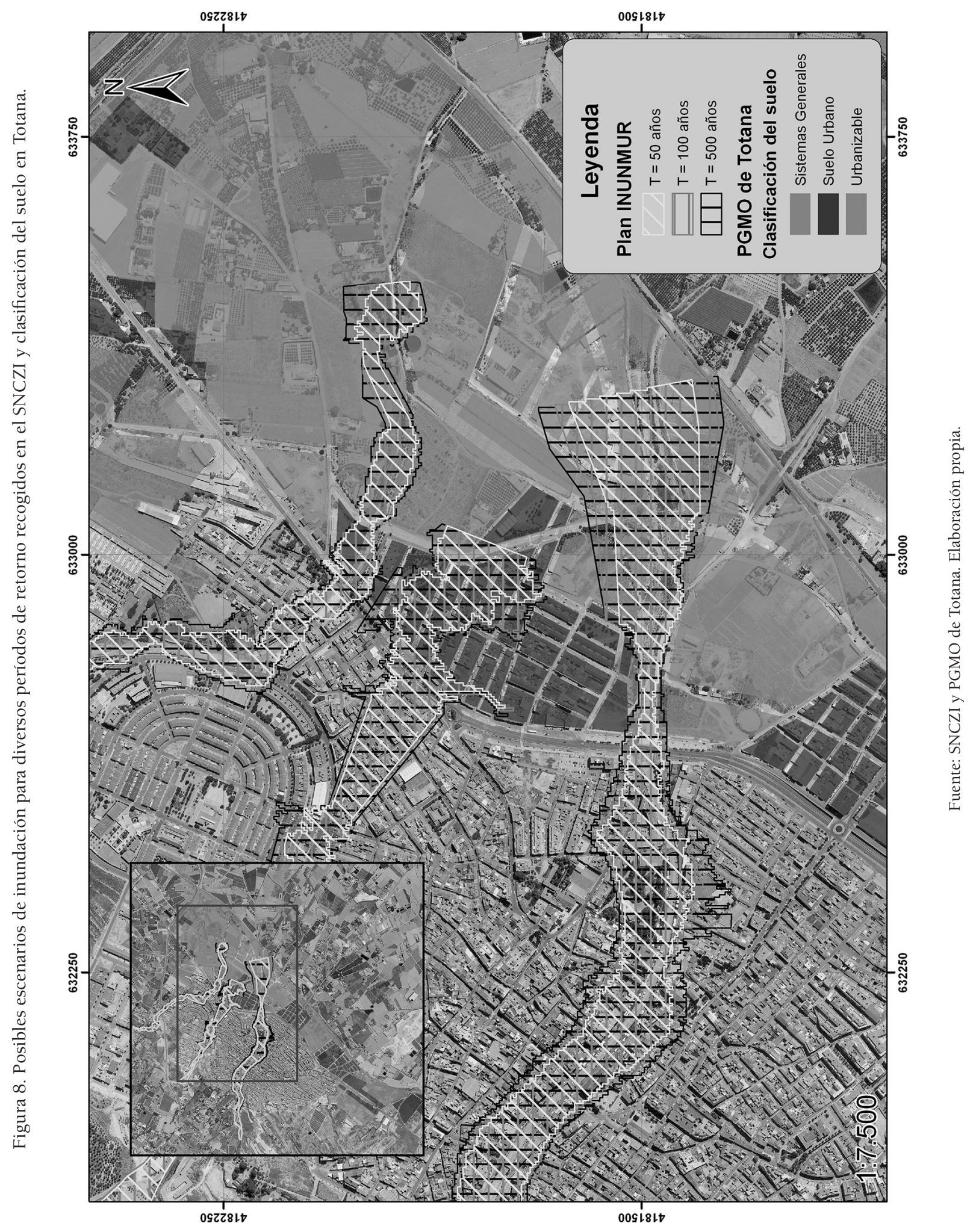




\section{BIBLIOGRAFÍA}

ANDERSON, M. B. y P. J. WOODROW (1989): Rising from the Ashes. Development Strategies in Times of Disaster. Ed. Westview Press, Boulder, Colorado (reeditado en 1998 por IT, Londres), 338 p.

AYALA-CARCEDO, F. J. (2002): "Introducción al análisis y gestión de riesgos", en Ayala-Carcedo FJ. y Olcina Cantos, J. (Eds.) Riesgos naturales. Editorial Ariel, S.A., Barcelona, pp. 133-145.

AYALA-CARCEDO, F. J., OLCINA CANTOS, J. y VILAPLANA FERNÁNDEZ, J.M. (2003): "Impacto económico y estrategias de mitigación de los riesgos naturales en España en el período 1990 - 2000", en Gerencia de riesgos y seguros. Fundación MAPFRE, nº 84, pp. 19-27.

BELTRÁN AGUIRRE, J.L. (2006): “Clasificación, categorización y calificación del suelo en la legislación autonómica comparada”, en Revista Jurídica de Navarra, n 41, pp. 81-112.

BOHLE, H.G. (2001): "Vulnerability and Criticality: Perspectives from Social Geography", en Newsletter of the International Human Dimensions Programme on Global Environmental Change, pp. 1-7.

CALVO GARCÍA-TORNEL, F. (1984): “La geografía de los riesgos”, en Geocrítica, no 54, pp. 1-37.

CALVO GARCÍA-TORNEL, F. (2001). Sociedades y Territorios en Riesgo. Ediciones Serbal, Barcelona, 186 p.

CAMARASA BELMONTE, A. M. (2002): "Crecidas e inundaciones", en Ayala-Carcedo FJ. y Olcina Cantos, J. (Eds.) Riesgos naturales. Editorial Ariel, S.A., Barcelona, pp. 859-879.

CAMARASA BELMONTE, M. y SORIANO GARCÍA, J. (2012): "Flood risk assessment and mapping in peri-urban Mediterranean environments using hydrogeomorphology. Application to ephemeral streams in the Valencia region (eastern Spain)", en Landscape and Urban Planning, Volume 104, Issue 2, pp. 189-200.

CANNON, T. (1994): "Vulnerability Analysis and the Explanation of 'Natural' Disasters", en Varley, A. (Ed.), Disasters, Development and Environment. Ed. John Wiley and Sons, Nueva York, pp. 13-30.

CONSORCIO DE COMPENSACIÓN DE SEGUROS (2008): La cobertura aseguradora de las catásterofes naturales. Diversidad de sistemas. Ed. Consorcio de Compensación de Seguros, Madrid, 218 p.

CUTTER, S. L., BORUFF, B. J. y SHIRLEY, W. L. (2003): "Social Vulnerability to Environmental Hazards", en Social Science Quarterly, vol. 84, n 2, pp. 242-261.

CRED (2013): Annual Disaster Statistical Review 2012: The numbers and trends. Universidad Católica de Louvain, Bruselas, Bélgica, 50 pp.

DARIO CARDONA, A. O. (1993): "Evaluación de la amenaza, la vulnerabilidad y el riesgo. Elementos para el Ordenamiento y la Planeación del Desarrollo", en "en Maskrey, A. (Ed.) Los desastres no son naturales. LA RED, Colombia, pp. 45-63.

DIEZ HERRERO, A. y PUJADAS FERRER, J. (2002): "Mapas de riesgos de inundaciones", en Ayala-Carcedo FJ. y Olcina Cantos, J. (Eds.) Riesgos naturales. Editorial Ariel, S.A., Barcelona, pp. 997-1012.

DIRECCIÓN GENERAL DE PROTECCIÓN CIVIL Y EMERGENCIAS. MINISTERIO DEL INTERIOR (2006). Catálogo Nacional de Inundaciones Históricas, CNIH. Fascículo 1. Madrid, recurso electrónico.

DIRECCIÓN GENERAL DE PROTECCIÓN CIVIL DE LA REGIÓN DE MURCIA (2007): Plan Especial de Protección Civil ante el riesgo de inundaciones de la Comunidad Autónoma de la Región de Murcia (Plan INUNMUR). Murcia, recurso electrónico.

FUENTES ZORITA, J.S. y CALVO GARCÍA-TORNEL, F. (1982): "La comarcalización de la Región de Murcia", en Estudios territoriales, n ${ }^{\circ}$ 7, pp. 89-125.

GONZÁLEZ AMUCHÁSTEGUI, M.J. (2002): "Procesos y riesgos naturales en la ordenación del territorio", en Euskonews n ${ }^{\circ} 153$.

GONZÁLEZ DE VALLEJO, L. (1988): "La importancia socioeconómica de los riesgos geológicos en España”, en Ayala-Carcedo F.J. y Durán, J.J. (Eds) Riesgos Geológicos. IGME, pp. 21-34.

IGME (2007). Estudio y cartografía de los peligros geológicos de las comarcas del Alto y Bajo Guadalentín de la Región de Murcia. Términos Municipales de Librilla, Alhama de Murcia, Totana, Aledo, Puerto Lumbreras y Lorca. Escala 1:50.000.IGME, Madrid, 237 p.

LLORENTE ISIDRO, M., DÍEZ-HERRERO, A. y LAÍN HUERTA, L. (2009): "Aplicaciones de los SIG al análisis y gestión del riesgo de inundaciones: avances recientes", en Cuadernos de la Sociedad Española de Ciencias Forestales, n 29, pp. 29-37.

MINISTERIO DE AGRICULTURA, ALIMENTACIÓN Y MEDIO AMBIENTE (2013). Sistema Nacional de Cartografía de Zonas Inundables, recurso electrónico.

OLCINA CANTOS, J. y GIMÉNEZ FERRER, J.M. (2004): "Riesgo de inundación en tierras alicantinas. Conceptos y métodos de trabajo", en Gil Olcina, A. Olcina Cantos, J. y Rico Amorós, A. M. (Eds.) Aguaceros, aguaduchos e inundaciones en áreas urbanas alicantinas. Publicaciones de la Universidad de Alicante, Alicante, pp. 21-36. 
OLCINA CANTOS, J. (2004): Riesgos de inundaciones y ordenación del territorio en la escala local. El papel del planeamiento urbano municipal, en Boletín de la Asociación de Geógrafos Españoles, $\mathrm{n}^{\circ} 37$ (monográfico "Agua y Ciudad"), pp. 49-84.

OLCINA CANTOS, J. (2007): Riesgo de inundaciones y ordenación del territorio en España. Fundación Instituto Euromediterráneo del Agua, Murcia, 381 p.

OLCINA CANTOS, J. (2010): "El tratamiento de los riesgos naturales en la planificación territorial de escala regional", en Papeles de Geografía, no 51-52, pp. 223-234

OLCINA CANTOS, J. (2012): "De los mapas de zonas afectadas a las cartografías de riesgo de inundación en España”, en Anales de Geografía, n 1, vol. 32, pp. 91-131.

ORTEGA ALBA, F. (1991): "Incertidumbre y riesgos naturales", en XII Congreso Nacional de Geografía. Valencia, pp. 99-108.

PARKER, D., TAPSELL, S., et al. (2009): "Deliverable 2.1. Relations between different types of social and economic vulnerability". Final draft report submitted to EU project Enhancing resilience of communities and territories facing natural and na-tech hazards (ENSURE), $89 \mathrm{pp}$.

PELEGRÍN GARRIDO, M.C. (2006): "Inundaciones históricas en Totana", en Cuadernos de la Santa, nº 8, pp. 201-204.

PÉREZ MORALES, A. (2008). Riesgo de inundación y políticas sobre el territorio en el sur de la Región de Murcia. Tesis Doctoral. Universidad de Murcia, Murcia, 659 p.

PÉREZ MORALES, A. (2010): "Valoración del riesgo de inundación en los instrumentos de ordenación del territorio de la Región de Murcia", en Papeles de Geografía, no 51-52, pp. 235-243.

PÉREZ MORALES, A. (2010): "El estudio de la política territorial como reflejo de la vulnerabilidad social ante el riesgo de inundaciones. El caos de Puerto Lumbreras, Sureste de España", en VI CIOT, Congreso Internacional de Ordenación del Territorio, Pamplona, pp. 222-233.

PÉREZ MORALES, A. (2012): "Estado actual de la cartografía de los riesgos de inundación y su aplicación en la ordenación del territorio. El caso de la Región de Murcia”, en Boletín de la Asociación de Geógrafos Españoles, n 58 , pp. 57-81.

PUJADAS, J. (2002): "Las inundaciones en España: impacto económico y gestión del riesgo", en Ayala-Carcedo F.J. y Olcina Cantos, J. (Eds.) Riesgos naturales. Editorial Ariel, S.A., Barcelona, pp. 859-879.

PUJADAS, J., PAZ, A. de, MARTURIÁ J. y VELASCO, E. (1997): “Cartografía de riesgos por inundación”, en Tecnoambiente, $\mathrm{n}^{\circ} 69$, pp. 54-59.

PULIDO BOSCH, A. (1993): "Las ramblas mediterráneas; condicionantes geomorfológicos e hidrológicos", en Cueto Romero, M. y Pallares Navarro, A. (Eds.) Regeneración de la cubierta vegetal. Las Ramblas Mediterráneas. Almería. Actas de la V y VI Aula de Ecología, Diputación de Almería, Instituto de Estudios Almerienses, pp. 131-140.

RIBERA MASGRAU, L. (2004): "Los mapas de riesgo de inundaciones: representación de la vulnerabilidad y aportación de las innovaciones tecnológicas", en Anales de Geografía, no 43, pp. 153-171.

ROMERO DÍAZ, A. (2007): "Las inundaciones", en Romero Díaz, A. y Alonso Sarriá, F (Eds.) Atlas Global de la Región de Murcia. Ed. La Verdad - CMM S.A. Murcia, pp. 250-260.

ROMERO DÍAZ, A. y MAURANDI GUIRADO, A. (2000): "Las inundaciones en la Cuenca del Segura en las dos últimas décadas del siglo XX. Actuaciones de prevención”, en Serie Geográfica, nº 9, pp. 93-119.

SÁENZ DE BURUAGA, G. (1980): "Ordenación territorial en la crisis actual”, en Ciudad y Territorio: Revista de Ciencia Urbana, $\mathrm{n}^{\mathrm{o}} 1$, pp. 17-24.

SAURÍ, D., RIBAS, A., LARA, A. y PAVÓN, D. (2010), "La percepción del riesgo de inundación: experiencias de aprendizaje en la Costa Brava", en Papeles de Geografía, no 51-52, pp. 269-278.

SMITH, K. Y PETLEY, D. N. (2009): Environmental Hazards. Assessing riks and reducing disaster. Ed. Routledge, $5^{\mathrm{a}}$ ed., London, $414 \mathrm{p}$.

WILCHES CHAUX, G. (1989). Desastres, ecologismo y formación profesional: herramientas para la crisis. Ed. Servicio Nacional de Aprendizaje (SENA), Popayán, 300 p.

WILCHES CHAUX, G. (1993): "La vulnerabilidad global", en Maskrey, A. (Ed.) Los desastres no son naturales. LA RED, Colombia, pp. 9-50.

WHITE, G. F., CALEF, W. C., HUDSON, J. W., MAYER, H. M., SHAEFFER, J. R. and VOLK, D. J. (1958): "Changes in Urban Occupance of Flood Plains in the United States", en Department of Geography Research Papers, $\mathrm{n}^{\circ}$ 57, 235 p.

WHITE, G. F. (1975): "La investigación de los riesgos naturales", en Chorley R (Ed.) Nuevas tendencias de la geografía. Ed. Instituto de Estudios de Administración Local, Madrid, pp. 281-315. 